\title{
Western Conceptualizations and Eastern Experience: A Cross-Cultural Study of Traumatic Stress Reactions Among Tibetan Refugees in India
}

\author{
Maaike A. Terheggen, ${ }^{1}$ Margaret S. Stroebe, ${ }^{1,2}$ and Rolf J. Kleber ${ }^{1}$
}

This study investigated the nature and impact of traumatic experiences among Tibetan refugees in India. It explored the applicability of western conceptualizations of reactions to traumatic events among this cultural group. A randomly selected sample of refugee camp students was assessed on measures of psychological and physical complaints, and on impact as well as severity of traumatic experiences. Respondents had experienced multiple traumatic events. The majority reported ongoing health problems. More than half demonstrated symptoms of intrusion-avoidance. Those with more traumatic experiences reported more symptoms of anxiety and depression (psychologically and, particularly, somatically manifested). Cultural differences in types of experienced traumatic events and manifestations of distress are discussed, as are implications for cross-cultural trauma research using western conceptualizations and instruments.

KEY WORDS: trauma; refugees; Tibet; cross-cultural; posttraumatic stress.

This study was designed to investigate the occurrence of traumatic experiences and consequent reactions of Tibetan refugees living in India, and to explore the extent to which the conceptualization of posttraumatic stress could be applied in a Tibetan refugee community. As such, this study provides an opportunity to investigate a nonwestern population of refugees who have continued to reside in a nonwestern milieu.

Research on posttrauma reactions in nonwestern groups has mostly been conducted within the United States and Western Europe, to assess and meet the needs

\footnotetext{
${ }^{1}$ Department of Clinical Psychology, Utrecht University, The Netherlands.

${ }^{2}$ To whom correspondence should be addressed at Department of Clinical Psychology, Utrecht University, P.O. Box 80.140, 3508 TC Utrecht, The Netherlands.
} 
of refugees from developing countries (see Kleber, Figley, \& Gersons, 1995). Differences in the prevalence of posttraumatic stress disorder (PTSD) across ethnic and cultural groups have been reported (Manson, 1985; Marsella, Friedman, Gerrity, \& Scurfield, 1996). Despite a concentration on Southeast Asian refugees (Abueg \& Chun, 1996; Sack, Seeley, \& Clarke, 1997; Ton-That, 1998), surprisingly few empirical studies have investigated posttrauma reactions in Asia itself. Studies of Asians living in the United States have yielded discrepant results, even within the same ethnic groups (Cambodians \& Vietnamese). There have been reports of high prevalences of PTSD (75-100\%: Carlson \& Rosser-Hogan, 1994; Kinzie et al., 1990), but also comparatively low prevalences (15-25\%: Abe, Zane, \& Chun, 1994; Kroll et al., 1989).

There are several conceptual issues related to cross-cultural trauma research. A significant reason for the discrepancies just mentioned could be that western diagnostic criteria are not applicable in nonwestern cultures (Knipscheer \& Kleber, 1999). Yet, out of 10 empirical studies known to the authors, only 2 questioned the validity of using western concepts and measurements in a nonwestern culture (Kinzie et al., 1994; Kroll et al., 1989).

A conceptual issue concerns the nature of traumatic stressors. These might not be the same in different cultural settings (Kleber et al., 1995). A standard checklist could underestimate the prevalence of extreme stressors if the list was not moderated according to the cultural values of the particular population (Manson, 1997). For example, in Tibet, the destruction of temples, monasteries, and other religious signs is an extremely stressful and frequently experienced traumatic event. If culturally defined stressors are ignored, the amount of stress experienced would be evaluated as lower than it actually is.

Furthermore, emotional distress experienced by members of a nonwestern culture may not be expressed in the same manner as in the West (Kleinman \& Cohen, 1997). Some symptoms may be displayed less, such as feelings of guilt among Tibetans, for which no Tibetan word even exists. In cases where feelings of guilt would be expected, feelings of shame are reported. Although different cultural meanings may underlie the presence or absence of symptoms, one must also be cautious in assuming that a particular constellation of symptoms-presumed to coincide with a disorder - can be explained by the same interpretation in different cultures (Bracken, Giller, \& Summerfield, 1995; cf. also Obeyesekere, 1985). Buddhism implies "that hopelessness lies in the nature of the world" (Obeysekere, 1985, p. 134). Consequently, a good Buddhist would display a symptom presentation of generalized feelings of hopelessness similar to depression. The acknowledgement of the all-pervasive presence of suffering in this world is almost "endorsed" by Buddhism. A "nondisorder" frame of reference for "depressive" symptoms is, therefore, present within Buddhist cultures.

There is also a complex association between the expression of distress and the occurrence of somatic symptoms in nonwestern cultures. It has been claimed 
that Asians display their distress in a more somatic way than do westerners (Kinzie et al., 1990; Mollica, Wyshak, de Marneffe, Khuon, \& Lavelle, 1987). There has been speculation about underlying causes and the meaning of apparent "somatic" expressions of distress (cf. Beiser \& Fleming, 1986; Mollica et al., 1987).

Against this background of complex methodological and conceptual issues and a limited body of cross-cultural trauma research, the task of investigating a particular nonwestern group experiencing extreme life events appears difficult. Nevertheless, there is urgency to increase scientific understanding, given the extremity of life events endured by certain nonwestern communities. Such is the situation for the Tibetan people, whose human rights have been violated since 1949. Even today, displays of Tibetan culture and the Tibetan Buddhist religion are still for the most part forbidden, and opponents of the new sovereignty still disappear, many others being imprisoned and tortured (e.g., Tibetan Review, 1995-99). The societal and religious leader of the Tibetans, the Dalai Lama, fled to India in 1959. One hundred thirty one thousand Tibetans followed him into exile in subsequent years. These refugees are likely to have been exposed to suppression and violence and to be troubled with severe emotional distress (Holtz, 1997).

Despite some documentation of extreme difficulties among Tibetan refugees (Mahmoudi, 1993; Rai, 1990) and indications of associated poststress reactions (Holtz, 1997; Servan-Schreiber, Lin, \& Birmaher, 1998), there has been very little scientific investigation of reactions to these extreme life events in this particular culture. Most relevant for the current investigation is the study by Holtz (1997) who studied the differences between Tibetan refugees who had experienced torture compared with those who had not. Torture survivors had significantly more symptoms of anxiety, but not of depression, than the nontortured cohort. Holtz's investigation (Holtz, 1997) did not extend to an examination of posttraumatic stress symptomatology. Furthermore, in concentrating only on the impact of torture, information on the range and severity of traumatic experiences was not collected.

The general interest of our study was to explore the validity and applicability of western concepts in nonwestern populations. We sought to investigate posttrauma reactions in a Tibetan refugee population that had recently fled from their home country to northern India. The key research questions were

1. Are traumatic experiences among these Tibetan people composed of similar categories of events as those that would be considered traumatic in the West?

2. After such events, do Tibetans show evidence of psychological symptoms, such as anxiety, depression, and the familiar PTSD symptoms of intrusion versus avoidance, similar to those found in the West? 


\section{Method}

\section{Participants}

Participants were inhabitants of an adult school (age 18-35) in a refugee camp in northern India. All newly arrived refugees within this age group are sent to this school, no selection criteria being imposed. Thus, the sample is representative of those fleeing from their home country within this age group.

Participants were randomly selected from the 220 students in the school's register by adding every third male on the list $(n=63)$, and thereafter every third female $(n=16)$, recruiting 79 students. The inequality in numbers between the sexes reflects the distribution at the school. If students were ill $(n=11)$ the next student on the school's list was recruited. Only three persons (all female) refused participation. The final sample was therefore 76 . Because of a few missing values on some of the (sub)scales, the number of cases in the statistical analyses did not always equal this total.

The age of participants ranged from 18 to $29(M=22, S D=2.5)$. They had left their homes 2.5 years -1 month prior to the study. Half of them had lived nomadically or had been farmers in Tibet; $20 \%$ had been students. Educational level varied: one-third had no education, one-third had more than 6 years. Current visits to the physician were rated by $50 \%$ as once or more a month and by $16 \%$ as once or more a week.

No further analyses using demographic data were conducted, because, despite apparent diversity in occupational and educational levels, the sample was quite homogeneous. None had had access to higher occupational positions. Previous education might not display ability: Schools in Tibet are not in reach for everyone (e.g., nomads). Although years of education varied, all were being educated at the same level in this refugee camp school. Thus, results are presented for the group as a whole.

\section{Procedure}

Questionnaires were first translated into Tibetan, followed by a backtranslation procedure (recommended by Brislin, Lonner, \& Thorndike, 1973). Using the translated questionnaires, an interview was conducted with three Tibetans who had good understanding of English, as suggested by Manson (1997) who described a structured inquiry to formulate cultural dimensions of PTSD. The aim here was to sensitize the investigator to Tibetans' reactions to trauma (i.e., to ensure that the items would make sense to Tibetans). The complete interview was scrutinized with each of the three persons; each question was examined for its comprehension and appropriateness. 
One of the authors (MT) and a Tibetan translator trusted by the participants (a Tibetan doctor), conducted the interviews in the school. Potential participants were told that the investigator (MT) was conducting research on health and personal experiences among school attendants. They were reassured about confidentiality and told that they were not obliged to answer questions that they did not wish to. Several participants could not read or write Tibetan; therefore, the translator read the introduction and the questions aloud. The options for answering questions were presented visually by displaying drawings of glasses of water, ranging from empty to full. Answer categories were written in Tibetan and English under the glasses. The answering of the given item was done in two ways. Participants were asked to point to their chosen answer (one of the glasses) and to give a verbal answer in Tibetan, which was then translated.

After the general introduction, participants completed the sociodemographic questions, the Posttraumatic Inventory (PTI), four additional questions to assess intrusion and avoidance symptomatology, and the Asian version of the Hopkins Symptom Checklist (HSCL-25).

\section{Instruments}

Traumatic experiences. The occurrence and the nature of traumatic experiences were measured using items from the Posttraumatic Inventory (PTI; Carlson \& Rosser-Hogan, 1994; Meinhardt, Tom, Tse, \& Yu, 1986), incorporating adaptations where these proved necessary. According to Carlson and Rosser-Hogan (1994), the original list was well tested and is useful for investigating traumatic experiences among refugees. The following adaptations were made, with local cultural considerations in mind: (1) The list was shortened, following informant feedback and pilot investigation, some items being collapsed (e.g., items that were the same except that they referred to "friends" vs. "relatives"). (2) Some items were left out because they were not relevant, (e.g., "Moving because of proximity to battles"). (3) Six items relating to traumatic events frequently happening to Tibetans were added. Sources for these were comments of the Dalai Lama (1990) and the Tibetan Review (1995). The items were as follows: being forbidden to live according to one's own religion, to speak one's own language, to live according to one's own culture, to have more than one child, the destruction of religious signs, and the disruption of family ties. In this way a 20 -item questionnaire was created.

Severity of experiences. To investigate the severity of the impact of traumatic experiences in a culturally sensitive manner, the procedure developed by Carlson and Rosser-Hogan (1994) was adopted. Eleven independent Tibetans were asked to rate the level of severity of each item of the PTI for a Tibetan. None of these persons took part in the main part of the study. Nine of them were students at the school, the other two were inhabitants of a nearby Tibetan village. Respondents were asked to read the list of events and to say how bad each item was for a Tibetan. A scale of 
0-100 was used (this was easy for these respondents because they were familiar with percentages). It was explained that $0-10$ was not bad, increasing to $90-100$, "very bad." By adding the average ratings for the items endorsed, weighted trauma scores for each item could be obtained, and the comparative severity calculated. An interrater reliability check yielded a standardized item alpha of .85 .

Intrusion and avoidance. The Impact of Event Scale (IES; cf. Horowitz, Wilner, \& Alvarez, 1979) could not be used as a measure of intrusion-avoidance among this sample. When attempts were made to translate the scale into Tibetan, it was found that the items became practically identical, and thus redundant. To rate the occurrence of intrusion and avoidance of thoughts relating to each one of the endorsed traumatic experiences, the following procedure was adopted. For each positively answered item on the PTI, four extra questions were asked. First, participants were asked when the event had happened, and whether the respondent had thought about it the previous week. Then, two items of the IES (Horowitz et al., 1979) were included, namely "Did you try not to think of it during the last seven days?" and "Did you think about it when you did not mean to during the last seven days?" Respondents were categorized into two groups: those who had no intrusionavoidance versus those who had intrusion or avoidance symptoms or both.

Symptoms of distress. Mollica et al. (1987) state that the HSCL-25 can be used universally to assess symptoms of distress. The list consists of anxiety (10 items) and depression (13 items) subscales from the original HSCL-58 (Derogatis et al., 1974). In the depression subscale two additional somatic symptoms ("poor appetite" and "difficulty falling or staying asleep") were added (Mollica et al., 1987), giving a total of 15 items. The HSCL total score can be used as a global measure of emotional distress (Mollica et al., 1987). The test-retest reliability of this instrument was found to be $r=.89$ for Southeast Asian refugees (Carlson \& Rosser-Hogan, 1994).

To measure physical complaints, the somatic subscale (three items) of the HSCL-58 was added. It made sense to add the two "depression" items that were originally classified as somatic by Mollica et al. (1987), to the somatic subscale. One more item was added to the somatic subscale (stomach pain), specifically for this investigation among Tibetans. The total somatic subscale was thus composed of six items. Cronbach's alpha was .67. This was considered adequate. No item could be deleted to obtain a higher alpha.

\section{Results}

\section{Traumatic Experiences}

Scores on the PTI indicated the nature and number of respondents' traumatic experiences. The mean number reported was three (total $=20)$. Scores ranged from 0 to 10 (reported by three persons). The order of frequency of reported 
traumatic experiences was as follows: destruction of religious signs, feeling one's life is in danger, relative or friend sent to prison, relative or friend tortured, having a relative or friend disappeared, and experiencing serious food shortage.

To obtain a more sensitive measure of the impact of these events, a severity index of traumatic events was computed, consisting of the average ratings of the 11 independent Tibetan raters (see Table 1). Following Carlson and Rosser-Hogan (1994), a weighted trauma score was obtained by adding the ratings of traumatic events endorsed by a respondent. The Pearson correlation between the number of traumatic events experienced by the respondents and the weighted trauma scores was $.97(p<.01)$. As total severity was so closely related to the number of traumatic events, further statistical analysis was limited to the number of events without consideration of relative severity.

\section{Emotional Distress}

Following the procedure adopted by Mollica et al. (1987) for the HSCL-25, a cutoff score of 1.75 on the 4 -point scale $(1=$ low to $4=$ high symptomatology) was

Table 1. Average Rating of Severity of Traumatic Experiences and Percentage of Respondents Endorsing That Item

\begin{tabular}{|c|c|c|c|}
\hline $\begin{array}{c}\text { Severity ranking } \\
\text { of events (most } \\
\text { severe-least severe) }\end{array}$ & Traumatic experience & $\begin{array}{c}\text { Average rating } \\
\text { by independent } \\
\text { assessors }(n=11)\end{array}$ & $\begin{array}{c}\text { Percentage of } \\
\text { sample endorsing } \\
\text { event }(n=76)\end{array}$ \\
\hline 1 & Destruction of religious signs & 95 & 43 \\
\hline 2 & Leaving home for political reasons & 88 & 16 \\
\hline 3 & $\begin{array}{l}\text { Being forbidden to live according } \\
\text { to one's own religion }\end{array}$ & 85 & 15 \\
\hline 4 & Feeling one's life is in danger & 82 & 32 \\
\hline 5 & $\begin{array}{l}\text { Being forbidden to live according } \\
\text { to one's own culture }\end{array}$ & 81 & 9 \\
\hline 6 & Relative or friend tortured & 81 & 28 \\
\hline 7 & Being assaulted or injured oneself & 80 & 13 \\
\hline 8 & Relatives or friends live in danger & 79 & 20 \\
\hline 9 & $\begin{array}{l}\text { Being forbidden to have more } \\
\text { than one child }\end{array}$ & 76 & 5 \\
\hline 10 & Being sent to prison & 76 & 25 \\
\hline 11 & Being tortured oneself & 75 & 12 \\
\hline 12 & $\begin{array}{l}\text { Relative or friend assaulted } \\
\text { or injured }\end{array}$ & 75 & 9 \\
\hline 13 & Relative or friend killed & 75 & 13 \\
\hline 14 & $\begin{array}{l}\text { Being forbidden to speak one's } \\
\text { own language }\end{array}$ & 74 & 3 \\
\hline 15 & Relative or friend sent to prison & 73 & 28 \\
\hline 16 & Experiencing food shortage & 69 & 26 \\
\hline 17 & Disruption of family ties & 64 & 0 \\
\hline 18 & Relative or friend disappeared & 60 & 26 \\
\hline 19 & Relative or friend moved in & 53 & 3 \\
\hline 20 & Leaving home for economic reasons & 28 & 3 \\
\hline
\end{tabular}


Table 2. Level of Distress According to the Hopkins Symptom Checklist $(N=76)$

\begin{tabular}{|c|c|c|c|c|}
\hline & HSCL & Anxiety & Depression & Somatic complaints \\
\hline Mean & 1.64 & 1.48 & 1.71 & 1.75 \\
\hline$S D$ & .29 & .40 & .39 & .32 \\
\hline Percentage above cutoff point & 35 & 25 & 42 & 50 \\
\hline
\end{tabular}

applied. Results are presented in Table 2. As can be seen, $35 \%$ of the participants reported substantial emotional distress; $25 \%$ showed substantial anxiety and $42 \%$ showed substantial depression. Half of the respondents showed somatic symptoms. To investigate the relationship between the number of reported traumatic experiences and the level of distress, correlations were computed. ${ }^{3}$ In general, more traumatic experiences were associated with higher levels of emotional distress, based on total scores for the Hopkins Symptom Checklist $(r=.25, p<.05)$. This result is due particularly to the anxiety subscale. The more the traumatic events experienced, the higher the scores on anxiety $(r=.28, p<.05)$. Correlations between the number of traumatic experiences and depression or somatic complaints were positive, but did not reach significance.

To establish whether those who experienced symptoms of intrusion and avoidance also had higher symptomatology, participants were divided into those who had intrusion-avoidance symptomatology versus those who did not. Separate scores for intrusion and avoidance and total intrusion-avoidance were calculated. $T$-Tests were conducted to examine patterns of emotional distress for those who had intrusion-avoidance symptomatology compared with those who did not, using the HSCL-25. Means for the total HSCL and for its three subscales are presented in Table 3. Respondents who reported intrusion had significantly higher scores on all subscales and the total HSCL, whereas those who reported avoidance scored significantly higher only on the somatic subscale (the correlation between intrusion and avoidance was .67). Respondents who reported intrusion or avoidance or both (i.e., the total impact score) had significantly higher scores on anxiety and depression. Their total symptomatology score was also higher. Differences between the two groups on somatic complaints did not reach significance, although respondents reporting intrusion-avoidance showed somewhat higher levels of somatic complaints.

\section{Somatic Manifestations of Distress}

Interestingly, it appeared that the three anxiety and three depression items of the HSCL that were endorsed most frequently by respondents were nearly all somatically phrased items (see Table 4), such as "faintness, dizziness, or weakness" (anxiety), or "feeling low in energy, slowed down" (depression). These somatically

\footnotetext{
${ }^{3}$ Because of sample size, no multivariate analyses were carried out.
} 
Table 3. Emotional Distress as a Function of Reported Intrusion and Avoidance

\begin{tabular}{lcccl}
\hline & \multicolumn{5}{c}{ Did not } \\
& Endorsed (\%) & endorse $(\%)$ & $t$ & \multicolumn{1}{c}{$d f$} \\
\hline Intrusion & & & & \\
$\quad$ Anxiety & 16.0 & 14.0 & $1.97^{*}$ & 59.52 \\
$\quad$ Depression & 26.5 & 23.8 & $2.69^{* *}$ & 63 \\
$\quad$ Somatic complaints & 9.0 & 8.1 & 1.64 & 56.20 \\
$\quad$ Total Hopkins Symptom Checklist & 51.2 & 46.1 & $2.34^{* *}$ & 57 \\
Avoidance & 15.7 & 14.8 & 0.82 & 62 \\
$\quad$ Anxiety & 26.5 & 24.7 & 1.54 & 63 \\
$\quad$ Depression & 7.9 & 8.9 & $-1.76^{*}$ & 58 \\
$\quad$ Somatic complaints & 49.7 & 48.8 & 0.38 & 57 \\
$\quad$ Total Hopkins Symptom Checklist & 15.9 & 14.0 & $1.87^{*}$ & 62 \\
Intrusion or avoidance or both & 26.7 & 23.2 & $3.68^{* * *}$ & 62.06 \\
$\quad$ Anxiety & 8.9 & 8.1 & 1.53 & 58 \\
$\quad$ Depression & 51.4 & 45.6 & $2.66^{* *}$ & 57 \\
$\quad$ Somatic complaints & & & \\
$\quad$ Total Hopkins Symptom Checklist & & &
\end{tabular}

Note. All participants reported exposure to at least one traumatic event.

$* p<.05$.

$* * p<.01$.

${ }^{* * *} p<.001$. All tests are one-tailed.

phrased items were originally designed to represent feelings of anxiety and depression, and not somatic complaints (cf. Mollica et al., 1987). To analyze the implications of the difference in type of phrasing, the total anxiety and depression subscales of the HSCL were divided into psychologically and somatically phrased items by three independent raters. An interrater reliability of $90 \%$ was obtained. Discrepancies were then discussed and agreement was reached on the final categorization (see Table 4). This additional analysis was included to shed more light on the somatization concern raised earlier in the paper. Analyses revealed that participants on average did indeed respond more affirmatively on items phrased in a somatic way, $t(73)=4.4, p<.001$ (anxiety items); $t(73)=6.95, p \leq .001$ (depression items).

\section{Discussion}

The Tibetan population studied in this investigation is a highly vulnerable group, given the enduring political situation in Tibet and the refugee status of these people in northern India. For many of the refugees, the experience of violence, terror, and destruction has not been limited to a single life event, but is composed of multiple, devastating occurrences in their recent histories. Coupled with this is ongoing uncertainty about the future.

Results showed that respondents had suffered multiple traumatic events. The concept of multiple traumatization (e.g., Becker, 1995) appears to be appropriate. It is important to establish the severity of the effect of these events. Those who had 
Table 4. Psychologically Versus Somatically Phrased HSCL Anxiety and Depression Items

\begin{tabular}{|c|c|c|c|}
\hline Subscale & Type of phrasing & Item & $\begin{array}{c}\text { Percentage } \\
\text { endorsement }\end{array}$ \\
\hline \multirow[t]{10}{*}{ Anxiety } & \multirow[t]{4}{*}{ Psychologically phrased } & Being suddenly scared for no reason & 19 \\
\hline & & Feeling fearful & 16 \\
\hline & & Feeling tense or keyed up & 27 \\
\hline & & Spells of terror or panic & 25 \\
\hline & \multirow[t]{6}{*}{ Somatically phrased } & Faintness, dizziness, or weakness & 59 \\
\hline & & Nervousness or shakiness inside & 43 \\
\hline & & Heart pounding or racing & 35 \\
\hline & & Trembling & 23 \\
\hline & & Headaches & 55 \\
\hline & & Feeling restless, not being able to sit still & 34 \\
\hline \multirow[t]{13}{*}{ Depression } & \multirow[t]{9}{*}{ Psychologically phrased } & Blaming oneself for things & 13 \\
\hline & & Feeling hopeless about the future & 28 \\
\hline & & Feeling blue & 59 \\
\hline & & Feeling lonely & 41 \\
\hline & & Thought of ending one's life & 8 \\
\hline & & Feeling trapped or caught & 31 \\
\hline & & Worrying too much about things & 75 \\
\hline & & Feeling no interest in things & 48 \\
\hline & & Feeling worthless & 27 \\
\hline & \multirow[t]{4}{*}{ Somatically phrased } & Feeling low in energy, slowed down & 67 \\
\hline & & Crying easily & 23 \\
\hline & & Loss of sexual interest or pleasure & 27 \\
\hline & & Feeling everything is an effort & 84 \\
\hline
\end{tabular}

suffered a larger number of traumatic events indeed suffered more psychological distress and higher levels of anxiety than those with fewer or no traumatic experiences. Symptoms of depression were not strongly correlated with the experience of traumatic events. However, this seems understandable given the circumstances of this cultural group and the fact that they were inhabitants of a refugee camp. The most frequently scored depression items (e.g., "worrying too much") could reflect refugee status. Following this line of reasoning, the high level of depressive symptoms is a result of circumstances common to all, and less a consequence of particular traumatic events. As suggested earlier, depressive symptom presentation among these Tibetans may also be strongly influenced by cultural or religious factors.

Was there evidence of posttraumatic symptomatology among these Tibetans? Our simple measure of intrusion-avoidance provides a useful indication. A significant difference was found on distress scores between those who suffered intrusiveavoidant symptomatology and those who did not. This could indicate the presence of posttraumatic stress disorder, although no standard PTSD assessment was conducted to confirm this. Not only was intrusion-avoidance significantly related to anxiety and depression but a trend was also found for somatic complaints.

Approximately a third (35\%) of the respondents met the criteria for "substantial emotional distress" set by Mollica et al. (1987). Twenty-five percent had 
excessive scores on anxiety, with an even higher proportion over the cutoff point for depression (42\%). In Holtz's study (Holtz, 1997), 29\% of the respondents met the criteria for substantial emotional distress, but only $14 \%$ scored over the cutoff point for depression whereas $41 \%$ reported symptoms of anxiety. These differences between the studies are difficult to interpret, but may have to do with sample differences (Holtz's respondents were predominantly nuns). The study of Bhutanese refugees in Nepal (Shrestha et al., 1998) revealed a similar pattern to ours, with nearly one third of the respondents reaching the cutoff point for substantial emotional distress. The proportion of respondents that endorsed anxiety was 38\%, whereas substantial symptoms of depression were reported by $20 \%$. Compared with Cambodian refugees (Carlson \& Rosser-Hogan, 1994), Tibetans experienced fewer traumatic events ( 3 compared with 14) and the general level of distress was also considerably lower (35\% compared with $86 \%$ over the cutoff point).

It became evident that events not included on a standard list of traumatic events are traumatic for Tibetans. There are remarkable differences compared with western experience in types and perceived severity of traumatic events. The three highest ranking traumatic events were connected with religion, not personal danger. Striking from a western point of view, is that "witnessing the destruction of religious signs" is not only considered to be traumatic, but that $95 \%$ of the raters endorsed this as the worst possible event that could happen to a Tibetan, and $43 \%$ had actually had this experience. Religion has always been an extremely dominant force in Tibetan culture and destruction by the ruling powers has been particularly focused on religious symbols, customs, and buildings.

A general recommendation would be to use standard tests, but to adapt these to be sensitive to the specific culture. It is possible that other studies in nonwestern cultures underestimate the number or the range of traumatic events or both of these. For example, in the Carlson and Rosser-Hogan (1994) study, a standard traumatic event checklist was used. Traumatic events relating to a personal sense of being in danger were ranked highest on the severity index. Neither this study nor the other two studies reviewed earlier (Holtz, 1997; Shresta et al., 1998) measured traumatic events related to religion, although the peoples concerned are known to have strong religious belief systems.

Cultural sensitivity is needed for the investigation of distress too. In our Tibetan study, respondents scored highest on somatically phrased items, and anxiety and depression items that were phrased in a somatic way were endorsed more frequently than those phrased in a psychological way. Interestingly, a similar type of symptom presentation was found by Carlson and Rosser-Hogan (1994) among their Cambodian sample. The somatically phrased items endorsed were almost the same in the two studies. Our tentative conclusion is that physical symptoms are a meaningful way of expressing distress.

There are evident limitations in the study design (e.g., not longitudinal; no PTSD interviews), but this Tibetan study provided unique information on trauma 
responses in a cultural perspective. Most cross-cultural studies of posttraumatic stress have been conducted with people already living in the West, for whom an acculturalisation process may have mediated the results (Furnham \& Bochner, 1986). In this study, refugees were living in northern India, within a Tibetan community, so participants were far less influenced by acculturalisation. A "no-trauma" control group could not be formed, as all Tibetans have lived under sovereignty and been confronted with violence. Nevertheless, meaningful comparisons could be made within the sample, between those who had experienced more versus fewer traumas.

To conclude, western conceptualizations of trauma and distress provide a useful basis for starting investigations in nonwestern cultures. We found evidence of traumatic reactions in a culture very different from western culture, and we found associated symptomatology that would be expected following such events. However, investigations need to be culturally sensitive to the identification of experiences that are to be classified as traumatic and to the expression of distress in relationship to these events-necessitating adaptation of the measures used to assess symptomatology.

\section{Acknowledgments}

The authors thank R. Brohm, A. Creszenci, M. Dubbink, F. van Holthoon, and E. Ketzer for their support in India. The Tibetans who participated in this study are thanked for their invaluable contribution, and W. Stroebe and H. Schut for comments on earlier versions.

\section{References}

Abe, J., Zane, N., \& Chun, K. (1994). Differential responses to trauma: Migration-related discriminants of posttraumatic stress disorder among southeast Asian refugees. Journal of Community Psychology, 22, 121-135.

Abueg, F. R., \& Chun, K. M. (1996). Traumatization stress among Asians and Asian Americans. In A. J. Marsella, M. J. Friedman, E. T Gerrity, \& R. M Scurfield (Eds.), Ethnocultural aspects of posttraumatic stress disorder: Issues, research and clinical applications (pp. 285-300). Washington, DC: American Psychological Association.

Becker, D. (1995). The deficiency of the concept of posttraumatic stress disorder when dealing with victims of human rights violations. In R. J. Kleber, Ch. R. Figley, \& B. P. R. Gersons (Eds.), Beyond trauma: Cultural and societal dynamics (pp. 99-110). New York: Plenum Press.

Beiser, M., \& Fleming, J. A. E. (1986). Measuring psychiatric disorder among southeast Asian refugees. Psychological Medicine, 16, 627-639.

Bracken, P. J., Giller, J. E., \& Summerfield, D. (1995). Psychological responses to war and atrocity: The limitations of current concepts. Social Science and Medicine, 40, 1073-1082.

Brislin, R. W., Lonner, W. J., \& Thorndike, R. M. (1973). Cross-cultural research methods. In R. T. Holt, \& J. E. Turner (Eds.), Comparative studies in behavioral sciences (pp. 32-58). New York: John Wiley and Sons.

Carlson, E., \& Rosser-Hogan, R. (1994). Cross-cultural response to trauma: A study of traumatic experiences and posttraumatic symptoms in Cambodian refugees. Journal of Traumatic Stress, 7, 43-58.

Dalai Lama. (1990). Freedom in exile. London: Hodder \& Stoughton.

Derogatis, L. R. et al. (1974). The Hopkins Symptom Checklist: A self-report symptom inventory. Behavioral Science, 19, 1-15. 
Furnham, A., \& Bochner, S. (1986). Culture shock: Psychological reactions to unfamiliar environments. London: Methuen.

Holtz, T. (1997). Refugee trauma versus torture trauma: A retrospective controlled cohort study of Tibetan refugees. Journal of Nervous and Mental Disease, 186, 24-34.

Horowitz, M., Wilner, N., \& Alvarez, W. (1979). Impact of event scale: A measure of subjective stress. Psychosomatic Medicine, 41, 209-218.

Kinzie, J. D., Boehnlein, J. K., Leung, P. K., Moore, L. J., Riley, C., \& Smith, D. (1990). The prevalence of posttraumatic stress disorder and its clinical significance among Southeast Asian refugees. American Journal of Psychiatry, 147, 913-917.

Kleber, R. J., Figley, Ch. R., \& Gersons, B. P. R (1995). (Eds.). Beyond trauma: Societal and cultural dimensions. New York: Plenum.

Kleinman, A., \& Cohen, A. (1997). Psychiatry's global challenge: An evolving crisis in the developing world signals the need for a better understanding of the links between culture and mental disorders. Scientific American, 3, 86-89.

Knipscheer, J. W., \& Kleber, R. J. (1999). Conceptual and methodological issues in mental health research with migrants in The Netherlands. Gedrag \& Gezondheid, 27.

Kroll, J., Habenicht, M., Mackenzie, T., Yang, M., Chan, S., Vang, T., Nguyen, T., Ly, M., Phommasouvanh, B., Nguyen, H., Vang, Y., Souvannasoth, L., \& Cabugo, R. (1989). Depression and posttraumatic stress disorder in Southeast Asian refugees. American Journal of Psychiatry, 146, $1592-1597$.

Mahmoudi, K. (1993). Refugee cross-cultural adjustment: Tibetans in India. International Journal of Intercultural Relations, 16, 17-32.

Manson, S. M. (1985). Culture and DSM-III: Implications for the diagnosis of mood and anxiety disorders. In A. Kleinman, \& B. Good, (Eds.), Culture and depression: Studies in the anthropology and cross-cultural psychiatry of affect and disorder (pp. 99-113). Berkeley: University of California Press.

Manson, S. M. (1997). Cross-cultural and multiethnic assessment of trauma. In J. P. Wilson \& T. M. Keane (Eds.), Assessing psychological trauma and PTSD (pp. 239-266). New York: Guilford.

Marsella, A. J., Friedman, M. J., Gerrity, E. T., \& Scurfield, R. M. (Eds.). (1996). Ethnocultural aspects of posttraumatic stress disorder: Issues, research and clinical applications. Washington: American Psychological Association.

Meinhardt, K., Tom, S., Tse, P., \& Yu, C. (1986). Southeast Asian refugees in the "Silicon Valley": The Asian health assessment project. Amerasia, 12, 43-65.

Mollica, R. F., Wyshak, G., de Marneffe, D., Khuon, F., \& Lavelle, J. (1987). Indochinese versions of the Hopkins Symptom Checklist-25: A screening instrument for the psychiatric care of refugees. American Journal of Psychiatry, 144, 497-500.

Obeyesekere, G. (1985). Depression, Buddhism and the work of culture in Sri Lanka. In A. Kleinman, \& B. Good (Eds.), Culture and depression: Studies in the anthropology and cross-cultural psychiatry of affect and disorder (pp. 134-152). Berkeley: University of California Press.

Rai, S. (1990). Perception of changes and difficulties by older and younger Tibetan refugees in India. Indian Journal of Current Psychological Research, 3, 90-94.

Sack, W. H., Seeley, J. R., \& Clarke, G. N. (1997). Does PTSD transcend cultural barriers? A study from the Khmer Adolescent Refugee Project. Journal of the Academy of Child Adolescent Psychiatry, $36,49-54$.

Servan-Schreiber, D., Lin, B., \& Birmaher, B. (1998). Prevalence of posttraumatic stress disorder and major depressive disorder in Tibetan refugee children. Journal of the American Academy of Child and Adolescent Psychiatry, 37, 874-879.

Shrestha, N. M., Sharma, B., Ommeren, M. van, Regmi, S., Makaju, R., Komproe, I., Shrestha, G. B., \& Jong, J. T. V. M. de (1998). Impact of torture on refugees displaced within the developing world: Symptomatology among Bhutanese refugees in Nepal. Journal of the American Medical Association, 280, 443-448.

Ton-That, N. (1998). Posttraumatic stress disorder in Asian refugees. Psychiatry and Clinical Neuroscience, 52 (Suppl.), S377-S379.

Wangyal, T. (Ed.). (1995-1999). Tibetan Review 30-34. 